\section{Antimicrobial Stewardship Programs: Engaging Project Managers to Increase Productivity}

To the Editor-In 2014, the state of California passed Senate Bill 1311, becoming the first state to mandate the judicious use of antimicrobial therapy through antimicrobial stewardship. ${ }^{1}$ The bill recommended assembly of a multidisciplinary antimicrobial stewardship committee including at minimum a physician and a pharmacist, and it called for hospitals to adopt and implement an antimicrobial stewardship policy in accordance with guidelines established by the federal government and professional organizations. ${ }^{1}$ In addition to a physician and a pharmacist, the Infectious Diseases Society of America guidelines for antimicrobial stewardship define an optimized antimicrobial stewardship program (ASP) as a multidisciplinary team encompassing specialists in infection prevention, clinical microbiology, hospital informatics, and hospital epidemiology. ${ }^{2}$ Neither organization, however, specifies the inclusion of a project manager, an individual who ensures optimal implementation of program objectives and overall management of the program. The ASP at UCLA Health serves 2 hospitals providing primary care to level V trauma care, with a combined capacity of 800 patient beds. In addition to the members endorsed by the Infectious Diseases Society of America, the UCLA ASP includes within its core structure a project manager, who is employed by the department of quality management services. Herein, we describe the role of the UCLA ASP project manager and how partnership with the quality department has added value in expanding and optimizing the scope of our ASP.

The UCLA ASP was implemented in July 2011 and comprises the following core team members: 2 infectious diseasetrained physicians, 3 infectious disease-trained pharmacists, the director of clinical microbiology, infection preventionists, a nurse coordinator for outpatient intravenous antimicrobial therapy, and a project manager. Our project manager has protected time to devote to ASP and is well-versed in lean process improvement methodology and data analytics. Responsibilities of the project manager include data generation and analysis, management of information systems used to track antimicrobial therapy audits, maintenance of educational resources including the ASP website, connection with other hospital departments and hospital administration, creation of deadlines and ensuring of forward momentum of projects, and organization and chairing of weekly ASP meetings. Additionally, the project manager facilitates annual program review and goal-setting while maintaining an active timeline of these goals to ensure completion. This is particularly important because the time of many ASP team members is not a "full-time equivalent" protected time devoted to ASP. By introducing a project manager, the burden of gathering, aggregating, and administratively reporting data is shifted from clinically focused team members to a data specialist. This allows clinicians and pharmacists to focus their time toward direct patient care activities.

As Section 1(d) of SB1311 states, California hospitals must report ASP activities to appropriate hospital committees undertaking clinical quality improvement activities. Many hospitals with an ASP do not receive direct compensation for ASP activities, and lack of funding has been identified as a primary barrier to initiating, maintaining, and expanding ASP services. ${ }^{3,4}$ As a member of the quality department, our project manager regularly interfaces with health-system administration, strategically placing her to convey the value of the ASP as it relates to hospital leadership planning, quality, and patient safety.

In addition to directly working with the ASP, a project manager can play an expanded role in other areas. For example, our project manager codesigned a program to expand employee seasonal influenza vaccination compliance, contributing to a tripling in the number of vaccinated staff within 2 years. ${ }^{5}$ She also worked with emergency preparedness teams to have UCLA Ronald Reagan Medical Center designated as an Ebola Care Center by the Centers for Disease Control and Prevention. As the project manager for the sepsis initiative, she worked with the ASP pharmacist to analyze the timeline of antibiotic administration and conduct time series analysis that directly led to a health system change of first-dose antibiotic delivery. Additionally, she created the Infection Prevention Leaders Academy, a 12-month training program for nurses on evidence-based practices and principles of infection prevention. ASP was included to discuss the topic of anti-infective resistance. The project manager is the principal communicator responsible for ensuring the ASP team is aware of changes and included during key institutional developments. The project manager's skill set and working relationships within the departments of quality, antimicrobial stewardship, and infection prevention offer an opportunity for alignment across programs, promoting cross-communication, and reducing the likelihood of redundant initiatives, thereby justifying continued full-time equivalent support.

The function of an ASP is to improve the quality of patient care by ensuring optimal treatment of infections, minimizing antimicrobial resistance and adverse events through their judicious use. Allocation of resources is a significant challenge facing many ASPs. Given that program validation focuses on cost savings rather than revenue generation, program justification is a constant concern of most 
ASP programs. Imperative to the success of program validation is the necessity of data collection and its analysis. The inclusion of a dedicated project manager allows for ongoing critical data analysis as well as interdisciplinary coordination across departments to improve the efficiency of the program while broadening the impact and scope of the ASP to improve patient care. We encourage other institutions interested in developing an ASP to reach out to their quality department for individuals trained in process improvement and program implementation.

\section{ACKNOWLEDGMENTS}

Financial support. None reported.

Potential conflicts of interest. All authors report no conflicts of interest relevant to this article.

\section{Brandy Bryant, MPH; ${ }^{1}$ Jennifer Curello, PharmD; Daniel Uslan, MD, FIDSA, FSHEA; ${ }^{3}$ Romney Humphries, $\mathrm{PhD}{ }^{4}$ Meganne Kanatani, PharmD; Zahra Kassamali, PharmD ${ }^{2,3}$}

Affiliations: 1. Department of Quality Management Services, UCLA Health System, Los Angeles, California; 2. Department of Pharmaceutical Services, UCLA Health System, Los Angeles, California; 3. Division of Infectious Diseases, David Geffen School of Medicine at University of California-Los Angeles (UCLA), Los Angeles, California; 4. Pathology \& Laboratory Medicine, David Geffen School of Medicine at University of California-Los Angeles (UCLA), Los Angeles, California.

Address correspondence to Brandy Bryant, MPH, Department of Quality Management Services, 924 Westwood Blvd, Ste 900, Los Angeles, CA 900242924 (bhamill@mednet.ucla.edu).

Infect Control Hosp Epidemiol 2016;37:739-740

(c) 2016 by The Society for Healthcare Epidemiology of America. All rights reserved. 0899-823X/2016/3706-0025. DOI: 10.1017/ice.2016.63

\section{REFERENCES}

1. California Senate Bill 1311. California Government website. http://leginfo.legislature.ca.gov/faces/billNavClient.xhtml?bill_ $\mathrm{id}=201320140$ SB1311. Published 2014. Accessed May 29, 2015.

2. Dellit TH, Owens RC, McGowan JE, et al. Infectious Diseases Society of America and the Society for Healthcare Epidemiology of America guidelines for developing an institutional program to enhance antimicrobial stewardship. Clin Infect Dis 2007;44: 159-177.

3. Johannsson B. Improving antimicrobial stewardship: the evolution of programmatic strategies and barriers. Infect Control Hosp Epidemiol 2011;32:367-374.

4. Trivedi K, Rosendberg J. The state of antimicrobial stewardship programs in California. Infect Control Hosp Epidemiol 2013; 34:379-384.

5. Bryant B, Sehl M, Uslan D. Seasonal influenza: increased healthcare worker vaccination does not decrease absenteeism. In: Program and abstracts of IDWeek 2015; October 7-11, 2015; San Diego, CA. Abstract 1910.

\section{Verbal Communication With Providers Improves Acceptance of Antimicrobial Stewardship Interventions}

To the Editor-Antibiotic-resistant bacteria are the greatest threat in modern medicine today. ${ }^{1}$ The Centers for Disease Control and Prevention (CDC) estimate that more than 2 million illnesses and 23,000 deaths are attributable to antibiotic-resistant bacteria. ${ }^{2}$ In recent years, collaborative efforts to mitigate the impact of these resistant organisms have arisen. As part of these efforts, the Society for Healthcare Epidemiology of America (SHEA) and the Infectious Diseases Society of America (IDSA) released a joint position statement emphasizing implementation of antimicrobial stewardship in all healthcare facilities. ${ }^{3}$ Additionally, the National Action Plan for Combating Antibiotic-resistant Bacteria, stemming from President Barack Obama's recent Executive Order, calls for a $20 \%$ reduction in inpatient antibiotic misuse by 2020 and mandates antimicrobial stewardship (AMS) in all acute-care hospitals. $^{4,5}$

While AMS is a multifaceted strategy, IDSA recommends 2 core approaches: prospective audit and feedback and formulary restriction. ${ }^{6}$ As part of prospective audit and feedback, AMS teams review patients receiving antibiotics for appropriateness of drug, duration, dose, and route. Proposed changes to antimicrobial regimens (interventions) are then relayed to the provider, often in the form of (1) a written or electronic notification placed in the patient's chart or electronic medical record (EMR) or (2) verbal communication, including face-to-face or telephone conversations.

Although overall acceptance of AMS interventions is well documented in the literature, acceptance according to different communication methods is unknown. ${ }^{7-10}$ We believe this gap in the current literature to be important and worthy of exploration because less effective methods of communication for AMS may be detrimental to patient care. In an effort to optimize patient care through our AMS services, we recently retrospectively evaluated the acceptance of all verbal and EMR interventions made by the AMS team at the Providence Veterans Affairs Medical Center between March 1, 2014, and February 28, 2015. Our AMS program has been existence since September 2012.

As part of prospective audit and feedback conducted every Monday through Friday, infectious diseases (ID) pharmacy fellows reviewed all patients admitted to the medical center receiving intravenous or oral antibiotics, antifungals, or antivirals. Appropriateness of antimicrobial selection, dose, duration, route, and indication were assessed according to current evidence in ID guidelines, primary literature, as well as our facility-specific antimicrobial guidebook. Interventions were organized into 11 categories: antimicrobial discontinuation, vancomycin dosing, drug dose or duration 